A N N A L E S Annales de Bretagne et des Pays de l'Ouest

Anjou. Maine. Poitou-Charente. Touraine

$109-2 \mid 2002$

Varia

\title{
L'intérieur des maisons rurales dans le Pays de Redon au XVIIIe siècle
}

Laurence Marquet

\section{OpenEdition}

Journals

Édition électronique

URL : http://journals.openedition.org/abpo/1607

DOI : $10.4000 /$ abpo. 1607

ISBN : 978-2-7535-1486-7

ISSN : 2108-6443

Éditeur

Presses universitaires de Rennes

Édition imprimée

Date de publication : 20 juin 2002

Pagination : 69-91

ISBN : 978-2-86847-742-2

ISSN : 0399-0826

Référence électronique

Laurence Marquet, "L'intérieur des maisons rurales dans le Pays de Redon au XVIIIe siècle ", Annales de Bretagne et des Pays de l'Ouest [En ligne], 109-2 | 2002, mis en ligne le 20 juin 2004, consulté le 20 avril 2019. URL : http://journals.openedition.org/abpo/1607 ; DOI : 10.4000/abpo.1607

(c) Presses universitaires de Rennes 


\title{
L'intérieur des maisons rurales dans le Pays de Redon au XVIII ${ }^{\mathrm{e}}$ siècle
}

\author{
Laurence MARQUET \\ Étudiante en maîtrise, Université de Rennes 2
}

Comprendre la vie quotidienne des paysans peut passer par l'étude d'un de leur principal cadre de vie : la maison ${ }^{1}$. Celle-ci recèle des objets et des effets témoignant de la façon dont ses habitants organisent leur vie quotidienne, de leurs habitudes et de leurs mentalités. Pour ce faire, les inventaires après décès sont indispensables. 691 actes $^{2}$ ont été dépouillés, concernant huit paroisses limitrophes de la ville de Redon ${ }^{3}$, pour la période allant de 1700 à 1790. L'objet de cette étude s'est porté essentiellement sur le mobilier, envisagé comme un moyen pour l'homme de répondre à ses besoins dans l'espace domestique de la maison. Les maisons rurales du Pays de Redon au XVIII ${ }^{\mathrm{e}}$ siècle se composent le plus souvent d'un rez-de-chaussée surmonté d'un grenier ${ }^{4}$. Ce modèle traditionnel est constitué d'une seule pièce à vivre appelée embas. Quelques rares maisons sont pluricellulaires, elles comportent une seconde pièce, à coté de la principale, parfois au-dessus. Ces intérieurs, caractérisés par la promiscuité, sont organisés autour de différents pôles. La cheminée est un pôle central car la vie domestique tourne autour de cette source de chaleur et de lumière qui permet également la cuisson. Les ouvertures constituent d'autres pôles; leurs dimensions réduites donnent à penser que la pièce est marquée par des zones

1. Cette étude est fondée sur un travail de recherche réalisé pour mon mémoire de maîtrise : Le mobilier rural paysan, l'organisation intérieure de l'habitat au XVII' siècle dans le Pays de Redon, mémoire de maîtrise, Université de Rennes 2, Annie Antoine (dir.), octobre 2001, 137 p. dactyl. [Lieu de consultation : CRHISco-Rennes 2].

2. Les 691 actes dépouillés correspondent à 676 inventaires après décès et 15 appositions de scellés.

3. La paroisse Notre-Dame englobe la zone urbaine de la ville de Redon et des villages (16,86\% des inventaires lui sont consacrés); les autres paroisses sont situées en zone rurale au proche de la ville : Bains (52,22\%); Avessac et son prieuré de Saint Nicolas $(14,35 \%)$; Celles éloignées de la ville : La paroisse d'Allaire, au travers du fief de SaintGorgon (5,62 \%); Renac (3,40 \%); Sixt (2,66 \%) et Saint-Just (1,33 \%).

4. HosDEZ, Juliette, L'habitat rural dans le Pays de Redon au xVII ' siècle, Mémoire de maîtrise, Université de Rennes 2, Annie Antoine (dir.), 1998, p. 102 [Lieu de consultation : CRHISCO-Rennes 2]. 
éclairées et des zones sombres. Les inventaires font souvent mention d'une " allée " ou de l'" entrée » de la maison, il s'agirait de l'espace proche de la porte d'entrée ou d'un vestibule. Comment le mobilier est-il disposé à l'intérieur de ces espaces et comment peut-on interpréter les rapports des habitants à leurs meubles? Entre le mobilier et son usager s'instaure en effet un rapport qui s'exprime au travers de la fonctionnalité, la gestualité, l'affectivité, l'économique, etc. L'objectif de cette étude est de dégager les principales caractéristiques de ce mobilier : formes, valeurs, fonctions (on peut par ailleurs se demander s'il est simplement fonctionnel ou s'il peut être ornemental). La distribution des meubles dans les maisons est étudiée comme indicateur du mode d'organisation de l'espace, les stratégies de rangement des objets permettent d'appréhender l'ordre (ou le désordre) régnant dans ces maisons et, au-delà, les mentalités de leurs habitants.

\section{Les lits}

Dans ces foyers paysans le meuble le plus indispensable et par conséquent le plus présent est le lit. Un seul foyer ne possède aucun lit, le paysan s'accommode alors d'" une balline, [d'] un vieux drap, [d'] un vieux berne ${ }^{5}$ " posé à même le sol. Toutes les autres demeures, visitées au moyen des 691 inventaires étudiés, comptent de un jusqu'à six lits. La répartition des lits dans les maisons s'opère de la façon suivante : $31,96 \%$ des foyers en possèdent un, $45,21 \%$ en ont deux, 18,11 \% en possèdent trois et $4,71 \%$ plus de trois. Il semblerait que le partage de la couche soit une pratique courante. Cependant au sein d'un même foyer, tous les dormeurs ne sont pas placés à la même enseigne.

\section{Les types de lits}

Les lits, désignés par les greffiers à l'aide de termes spécifiques, ont été répartis en quatre groupes. Le premier recense 1,3\% des couches, ils sont destinés à des dormeurs mal lotis puisqu'ils se composent d'éléments de literie disposés à même le sol. Ce sont, dans tous les cas, des lits secondaires. Entrent dans ce groupe d'autres substituts du lit : le coffre et le banccoffre servant de châssis à l'intérieur duquel est posée une literie. Un inventaire nous révèle " un mauvais coffre servant de lit d'enfant ${ }^{6}$ ", un autre " un petit banc-coffre éffoncé sur lequel une petite couette de balle, un vieux linceul de toile réparon ${ }^{7}$ ». En toute hypothèse, ce type de lit revient aux enfants, sûrement en bas âge.

5. Arch. dép. d'Ille-et-Vilaine, 4B 4291, Jan Fougère, 10 juin 1755, Journalier, Avessac, 2 livres.

6. Arch. dép. d'Ille-et-Vilaine, 4B 4150, Jean Lambert, 14 février 1766, Activité?, Saint Gorgon, 465 livres.

7. Arch. dép. d'Ille-et-Vilaine, 4B 4344, Jacquette Siloret, 29 octobre 1750, Veuve de Julien Derune, Renac, 949 livres. 
Le second type rassemble de vrais lits, constitués d'un cadre de bois supportant la literie. Ils représentent la grande majorité des lits rencontrés dans ces intérieurs puisqu'ils constituent 93,81\% de la totalité des lits répertoriés. Ils sont désignés par trois termes : châlit, couchette et lit. Il semblerait qu'il y ait une différence entre ces trois lits car au sein d'un même foyer ces termes coexistent. La distinction se fait peut-être par rapport à leur aspect, mais nous avons très peu de renseignements à ce sujet. De plus les greffiers font des confusions entre ces meubles, puisque lors d'une apposition de scellés le greffier note une couchette qui devient un châlit lors de la rédaction de l'inventaire. Le laconisme des sources sur la description de ces bois de lits ne permet pas de visualiser distinctement leurs formes. D'une manière générale, il existe des lits de tailles différentes : certains sont qualifiés de petit, grand, bas, demi. Ces variantes sont à prendre en compte pour l'attribution du lit : le lit petit, demi ou bas reviendrait aux enfants. D'autres renseignements sur leur état, avec les adjectifs vieux, mauvais et, plus rarement, neuf, indiquent que les paysans s'accommodent d'un meuble usé et que ces marques d'âge n'impliquent pas le renouvellement. Quelques indications supplémentaires sont d'interprétation délicate, par exemple " un châlit sans pied "; cette remarque signifie peut-être que cette couche sort de l'ordinaire alors que ce type de lit serait habituellement surélevé par rapport au sol. Dans un autre inventaire, un châlit est prisé sans devant. Le devant du lit est la façade par laquelle les dormeurs entrent dans le lit et donc celle qui est exposée aux regards. Ainsi un devant aurait été posé à la verticale du cadre de bois. Ce qui renverrait au lit dit mi-clos. Certains de ces lits sont dits "à la mode de la campagne " ou encore " à l'ancienne mode ", ce qui renvoie sûrement à un style particulier, peut-être démodé, conservé dans les campagnes.

Le troisième groupe concerne les lits spécifiquement destinés aux enfants, soit $1,28 \%$. Ils se répartissent en $0,6 \%$ de bers $^{8}$ et $0,68 \%$ de lits d'enfant, ils sont donc extrêmement rares, encore ne sont-ils repérés que dans la deuxième moitié du XVIII ${ }^{\mathrm{e}}$ siècle. Cette rareté est-elle due au fait que l'inventaire intervient à un âge avancé de la vie du défunt alors qu'il n'a plus d'enfants en bas âge sous son toit? ou plus simplement les parents s'accommodent-ils de ce qu'ils ont (un coffre par exemple)? ou bien encore le petit enfant dort-il avec ses parents?

Enfin le dernier groupe, en effectif moindre, correspond à des meubles de repos plus élaborés. Il s'agit de 1,28 \% de lits clos, recensés surtout dans la première moitié du siècle. La présence du lit clos dans cette partie de la Bretagne est ainsi attestée même s'il ne représente pas le modèle traditionnel. Sont également présents 2,34 \% de lits à quenouilles ou à colonnes, des lits qui ont nécessité plus de travail et qui sont plus chers. Ce sont des lits détenus par des paysans aisés, vivant dans des paroisses proches de la ville de Redon.

8. Terme désignant un berceau. 


\section{La disposition des lits}

Autour du lit, les paysans ont accolé d'autres meubles. Ainsi au-devant du lit, c'est-à-dire au long, est le plus souvent placée une maie, quelquefois un banc-coffre ou plus rarement un banc. La maie est privilégiée car étant un meuble bas, elle est pratique pour grimper dans le lit auquel elle sert de marchepied. Nous apprenons ainsi que les lits étaient plus hauts qu'actuellement afin d'éloigner le corps du froid et de l'humidité du sol. Au pied du lit est souvent disposé un coffre. L'aménagement des lits dans la maison peut nous renseigner sur les mentalités de ces paysans. Quand la pièce unique compte plusieurs lits comment les occupants des lieux les disposent-ils? Un exemple parmi tant d'autres montre " une couchette avec une couette de plume, deux draps de lit, deux bernes, proche de la fenêtre, prisée 45 livres; une couchette avec une petite balline avec des rideaux de toile, au fond de la maison, prisée 45 livres $^{9}$ ". La pratique est de disperser les lits dans l'espace domestique et non de les accoler les uns aux autres. Quels sont les emplacements privilégiés pour placer les lits? Une abondance d'exemples nous indique que ce sont les abords de la cheminée, mais aussi les ouvertures (fenêtre et portes). Il arrive également qu'un lit soit placé dans l'allée ou l'entrée de la maison, ceci est sûrement un moyen de désencombrer la pièce commune. Quand la maison comporte des pièces secondaires, on constate la présence de lits dans celles-ci. Elle est aussi constatée dans les bâtiments annexes, étable, greniers ou hangars, granges et celliers. Ces lits servent car ils sont garnis d'une literie. Donc la distribution des lits dans la maison voire dans les dépendances répond au problème du manque de place, et leur dispersion dans l'espace domestique témoigne d'une certaine recherche d'intimité.

\section{Le confort des lits}

Est-ce que ces paysans dorment dans un lit confortable? Les inventaires ont décrit les lits avec leurs garnitures, dont la literie. Le modèle traditionnel est le suivant : sur le bois de lit, est posée une couette, plus rarement une paillasse, en guise de sommier voire de matelas. À l'époque la couette désigne " un grand coussin qui sert à recevoir le poids du corps ${ }^{10}$ ". En fait, elle sert de matelas. Elle est remplie de plume ou de balle, mais c'est la plume, plus confortable, qui a été rencontrée le plus souvent ${ }^{11}$. Il semble que les paysans se délassent sur une literie quelque peu rude et qui offre peu de chaleur. Six lits ont révélé contenir deux couettes posées l'une sur l'autre, sûrement pour la recherche de la souplesse. Outre une couette,

9. Arch. dép. d'Ille-et-Vilaine, 4B 4170, Marie Geffray, 10 avril 1787, Veuve de Claude Guérif, Avessac, 416 livres 2 sols.

10. REYNIES, Nicole de, Le mobilier domestique, vocabulaire typologique, Paris, Imprimerie Nationale, tome 1.

11. Pour le XVIII ${ }^{\text {e }}$ siècle, $68,96 \%$ des couettes sont de plume (nous ne savons pas de quel volatile provient la plume); 31,04\% sont de balle (enveloppe de l'épi d'avoine). 
$20,43 \%$ des lits contenaient une balline ${ }^{12}$. Sur ces couettes sont disposés des draps. Le terme de linceul (toile de lin) revient dans les inventaires jusqu'aux années 1760, puis il cède la place au terme " drap de lit " ou tout simplement "drap ». 93,10\% des lits garnis possèdent deux draps. Reste à savoir comment ils sont disposés dans le lit. La réponse la plus évidente serait de dire qu'un drap est placé au-dessous et un autre au-dessus du corps pendant le sommeil. Ils sont faits de toile de brin réparon et de toile de réparon, c'est-à-dire de fil de lin. Le premier matériau est un fil plus grossier et plus rude et de qualité inférieure par rapport à la toile de brin qui est plus fine. Quant aux dimensions de ces draps, les inventaires sont restés muets. Un seul cas a révélé " un morceau de toile en forme de drap de lit d'environ seize empans de toile ${ }^{13}$ " soit un peu plus de $3,50 \mathrm{~m}$, mais estce un cas ordinaire? En ce qui concerne la réserve de draps, certains paysans en remplissent leurs meubles de rangement mais, dans la plupart des cas, la réserve est minime, ce qui laisse à penser que les draps ne sont pas changés régulièrement. Ensuite est posé un berne en guise de couverture : c'est une toile rembourrée. Mais les inventaires ne précisent pas le matériau utilisé et par conséquent il est difficile de savoir si ce berne procure de la chaleur. Cependant on remarque que $41,8 \%$ de lits comptent un berne et $39,8 \%$ en comptent deux : l'accumulation de bernes, pour remédier au froid, est assez fréquente. Les éléments de la literie sont le plus souvent qualifiés de vieux ou de mauvais, comme dans cet exemple où tous les éléments de la couche sont en mauvais état : "Un bois de couchette garni en dedans d'une vieille couette de plume, un mauvais oreiller de plume, deux mauvais linceuls de toile de brin réparon et un mauvais berne, prisé 3 livres 10 sols $^{14}$. " À ces indices nous pouvons penser que les paysans ne changeaient que rarement leur literie même quand celle-ci était usée.

Le lit traditionnel de la région de Redon semble donc peu satisfaisant, tant du point de vue de l'hygiène que de la chaleur. Il témoigne d'un confort rudimentaire, le sommeil semble devoir être quelque peu gêné par le manque de souplesse de la couche et le contact avec des matières qui seraient irritantes pour nous. Cependant une infime partie de paysans, aisés, connaît de nouveaux éléments de confort. La couverture apparaît sur quelques rares lits (4,97\% des lits inventoriés) où elle remplace le berne. L'accumulation de couvertures est chose rare. La couleur de cette dernière est un élément important. Le vert domine, à hauteur de 70,96\% des cas pour lesquels une couleur était indiquée. Les autres sont le blanc $(12,9 \%)$, le rouge $(6,45 \%)$, le jaune $(3,22 \%)$, et le bleu $(3,22 \%)$. Oreiller et traversin ne font qu'une apparition très discrète dans les lits, mais ils sont révélateurs de la recherche d'un mieux être matériel. L'oreiller est présent dans $7,19 \%$ de lits, il est rempli de plume ou de balle. Dans $75 \%$ des cas

12. Ce serait une paillasse remplie de balle pour les petits enfants, peut être plus petite que la couette.

13. Arch. dép. d'Ille-et-Vilaine, 4B Julien Derune, 23 octobre 1745, Renac, 84 livres 16 sols. 14. Arch. dép. d'Ille-et-Vilaine, 4B 4375, Jan Cheval, 28 mai 1759, Activité?, Sixt, 85 livres 8 sols. 
mentionnant cet élément, il n'y en a qu'un. Le traversin est présent dans $0,77 \%$ des lits, il est rempli lui aussi soit de plume soit de balle. Cet élément est prisé essentiellement chez des paysans de la paroisse de Notre-Dame et de Bains, c'est-à-dire dans une zone proche de la ville, témoignant sans doute d'une influence du mode de vie urbain. La literie des bers semble identique à celle des lits ainsi que l'indique cet exemple : " un ber avec une balline, un berne ${ }^{15}$ ".

Les inventaires font apparaître des lits clôturés de rideaux (12,08 \% des lits). Le tableau suivant témoigne de la répartition du nombre de ces lits par foyer.

Tableau 1 - Nombre de lits clôturés par foyer

\begin{tabular}{|lr|}
\hline Un seul lit clôturé & $58,19 \%$ \\
Plusieurs mais pas tous les lits & $13,93 \%$ \\
Tous les lits & $27,86 \%$ \\
\hline
\end{tabular}

Le rideau servait non seulement à remédier au froid mais surtout à rechercher une certaine intimité, car on observe que lorsque la maison dispose de pièces annexes et qu'un lit y est relégué il est dépourvu de rideaux. Diverses matières sont utilisées pour faire ces rideaux. Le matériau le plus courant est la toile de lin, la même que celle des draps. Le lin étant une ressource locale importante, les paysans l'utilisent pour la confection de diverses pièces de linge. À côté de cette grosse toile, les tissus en laine sont également utilisés comme la serge, la tiretaine et le droguet. Même s'ils sont rares, des " rideaux en poil de chien ${ }^{16}$ " ont été recensés. La couleur qui domine pour les rideaux est le vert $(69,33 \%$ des rideaux dont la couleur était précisée), puis le jaune (9,33\%), le brun (8,33\%), le rouge (5,55\%), le blanc (2,77 \%); nous avons aussi rencontré la mention " des rideaux de toile teinte " (4,10\% d'entre eux). Aucune mention n'est faite d'une quelconque pièce (tringle) qui les attacherait au lit; nous pouvons donc penser que les rideaux sont fixes et non coulissants. En ce qui concerne leur nombre et le côté du lit sur lequel ils sont placés, ceci est difficile à préciser puisque des exemples divers proposent des dispositions différentes : " un bois de couchette garni en devant et au pied de rideaux de serge ${ }^{17}$ "; " un bois de couchette garni en devant de deux petits rideaux ${ }^{18}$ "; " un bois de couchette garni autour de vieux rideaux de toile ${ }^{19}$ ". Ces exemples nous amènent à pen-

15. Arch. dép. d’Ille-et-Vilaine, 4B 4137, Jan Mahé, 22 décembre 1753, Laboureur, Bains, 209 livres 5 sols.

16. Arch. dép. d'Ille-et-Vilaine, 4B 4345, Denise Poligné, 13 avril 1785, Veuve, Saint Just, 140 livres 12 sols.

17. Arch. dép. d'Ille-et-Vilaine, 4B 4344, Pierre Debray, 14 août 1753, Activité?, Renac, 825 livres 10 sols.

18. Arch. dép. d'Ille-et-Vilaine, 4B 4359, Bertrand Durand, 22 avril 1756, Activité?, Renac, 128 livres 10 sols.

19. Arch. dép. d'Ille-et-Vilaine, 4B 4344, Guillaume Baudu, $1^{\mathrm{er}}$ mars 1749, Activité?, Renac, 212 livres 17 sols. 
ser que le nombre et l'emplacement des rideaux dépendent de la disposition du lit; dans cette région, le cas le plus fréquent est celui du lit de coin, il est alors clôturé sur le devant, parfois aussi au pied.

\section{Le prix des lits}

Le lit est primordial sur le plan de la symbolique, mais il l'est aussi sur le plan matériel : il est la pièce la plus coûteuse du mobilier. Les estimations extrêmes vont de 15 sols $^{20}$ jusqu'à 66 livres $^{21}$. Plus de la moitié des lits sont estimés à moins de 10 livres mais, au fil du siècle, le nombre de lit dont la valeur est supérieure à 20 livres augmente. À la lecture des inventaires nous constatons que, dans un intérieur possédant plusieurs lits, chacun a une valeur différente. Un des lits peut coûter plus de 20 livres et les autres moins de 10 livres. Une certaine hiérarchie des dormeurs s'exprime donc ainsi. Pour mieux comprendre ces différences, il faut tenir compte de la manière dont est réalisée l'estimation : quand un greffier estime un lit il évalue généralement le tout (le bois, les éléments de garniture, les rideaux). Quelques rares cas donnent une prisée de chacun des éléments : «Un bois de lit à la mode de la campagne, prisé 1 livre; un autre bois de lit à la mode de la campagne prisé 4 livres; [et leur contenu respectif] une couette de plume 10 livres; une autre couette de plume 8 livres ${ }^{22}$. " Dans un autre lit, les éléments de la literie sont prisés individuellement : " un berne, 2 livres; une vieille couette de plume, 4 livres; deux linceuls de toile de brin réparon, 2 livres 10 sols ${ }^{23}$ "; ou encore " un oreiller de plume 12 livres, une couverture de laine verte 5 livres ${ }^{24}$ ". Les éléments de la literie coûtent donc beaucoup plus cher que le cadre de bois; nous comprenons pourquoi les couvertures et oreillers n'investissent que rarement les lits. L'attribution du lit le plus coûteux, le mieux garni, clôturé, revient sûrement au couple conjugal. La valeur du lit représente en moyenne 51,43\% de la valeur totale du mobilier, et 10,17 \% de l'ensemble des biens. Le lit est bien le luxe du paysan. Pour certains c'est leur unique richesse. C'est la raison pour laquelle une grande partie des inventaires commence par la prisée des lits. Sur le plan symbolique, le lit est également d'une très grande importance dans ces maisons. Tout d'abord, la principale couleur que nous rencontrons est le vert. Une couleur qui reflète les valeurs familiales comme la joie et la fécondité. En y ajoutant les nouveaux tissus et nouveaux éléments

20. Pour une couchette avec une mauvaise balline : Arch. dép. d'Ille-et-Vilaine, 4B 4157, Michelle Chesnais, 28 juin 1773, Activité?, Notre Dame, 100 livres 19 sols.

21. Pour un lit à la mode de la campagne avec de mauvais rideaux, une balline, une couette de plume, un oreiller, deux draps, un berne : Arch. dép. d'Ille-et-Vilaine, 4B 4165, Alain Riaud, 25 août 1781, Laboureur, Notre Dame, 1052 livres 5 sols.

22. Arch. dép. d'Ille-et-Vilaine, 4B 4095, Renée Lejeune, 20 août 1711, Veuve, Avessac, 372 livres 3 sols.

23. Arch. dép. d'Ille-et-Vilaine, 4B 4344, Fabienne Jolly, 15 mars 1756, Activité?, Sixt, 445 livres 14 sols 6 deniers.

24. Arch. dép. d'Ille-et-Vilaine, 4B 4102, Vincent Hurel, 30 avril 1718, Activité?, Bains, 169 livres 16 sols. 
du confort, les paysans adoptent les références des élites de cette société hiérarchique. Cette appropriation d'un certain goût du beau et de la décoration donne de la gaieté à ces pièces sombres, mais nous ne le trouvons que dans un très petit nombre de foyers paysans.

Les paysans du pays de Redon dorment donc dans un vrai lit mais dont le confort est sûrement insatisfaisant. Ce qui saute aux yeux c'est la hiérarchisation de ces dormeurs. Dans un même intérieur, tous ne sont pas placés à la même enseigne devant le confort de la literie, l'emplacement (près de la cheminée ou dans l'étable), l'intimité (lit clôturé).

\section{La table}

Selon Anne Fillon ${ }^{25}$, la table serait une création moderne de la fin du XVII ${ }^{\mathrm{e}}$ siècle environ. La première question qui vient alors à l'esprit est de savoir si ce meuble s'est ensuite largement répandu au cours du XVIII ${ }^{\mathrm{e}}$ siècle. La première constatation est celle d'une absence de table dans 17,67 \% des foyers paysans de la région de Redon. Cette absence croît tout au long de la période. Les paysans auraient-ils recours à un substitut, comme le coffre? Mais " un coffre servant de table 1 livre 10 sols $^{26}$ ", " un vieux coffre servant de table 1 livre $^{27}$ " et un mauvais coffre servant de table ${ }^{28}$ " sont les seuls exemples rencontrés qui évoquent cette pratique.

Dans les autres foyers possédant des tables, combien en comptonsnous? Sur l'ensemble de la période, 75,14 \% des foyers en possèdent une seule; ce meuble est donc connu par une grande partie des paysans au XVIIIe siècle. Mais en regardant de plus près, nous constatons que ces intérieurs à une seule table diminuent à mesure que le siècle avance. Comment expliquer ce phénomène? Sa valeur est estimée le plus fréquemment entre 1 et 5 livres, elle a donc un coût relativement peu élevé. De plus les greffiers qualifient souvent les tables de "peu de valeur " et elles sont alors prisées autour d'une livre. Cette valeur reste stable sur toute la période : 78,48\% d'entre elles sont estimées entre 1 et 5 livres de 1700 à 1730, et 73,11 \% d'entre elles de 1761 à 1790. Par conséquent, le prix n'a pas d'incidence sur le recul de la table. Est-ce un problème de fiabilité des sources? La comparaison entre l'apposition de scellés et l'inventaire d'un même défunt peut révéler des disparitions d'effets, comme dans l'exemple suivant : alors que dans le premier acte " une table vide " est mentionnée, dans l'inventaire la table ne figure plus dans la liste des biens. Que s'est-il passé entre temps?

25. FILlon, Anne, "Comme on fait son lit on se couche", Populations et cultures. Études réunies en l'honneur de François Lebrun, Les Amis de François Lebrun, Rennes, Université Rennes 2/Institut Culturel de Bretagne, 1989.

26. Arch. dép. d’Ille-et-Vilaine, 4B 4292, Françoise Mousset, 24 février 1768, Activité?, Prieuré Saint Nicolas, 414 livres 17 sols.

27. Arch. dép. d'Ille-et-Vilaine, 4B 4147, Julien Macé, 31 décembre 1763, Laboureur, Bains, 185 livres 7 sols.

28. Arch. dép. d'Ille-et-Vilaine, 4B 4151, Jean Danto, 21 mars 1767, Activité?, Bains, 169 livres 10 sols. 
Aucune déclaration n'a été faite. Dernière hypothèse, certains paysans ne voient peut-être pas la table comme un meuble à utilité alimentaire et certains la remplacent par un coffre. Cela pose la question des fonctions et de l'usage de la table à cette époque dans cette région de Bretagne.

Les officiers de la justice seigneuriale ont l'habitude de commencer par l'estimation des biens de la pièce unique ou principale : la table y trouve place. Elle est donc installée dans la pièce commune. Nous remarquons aussi que, quand un foyer compte deux tables, l'une d'elle est placée dans l'allée ou dans l'entrée de la maison. Son emplacement est parfois suggéré par sa proximité avec le lit ${ }^{29}$. Il faut aussi noter que le meuble marchepied qui est disposé près du lit peut servir autant à monter dans le lit qu'à s'asseoir autour de la table. Cette imbrication n'a vraisemblablement pas d'autre but que de gagner et de libérer de l'espace. Nous pouvons penser que ces paysans placent le plus souvent la table dans un espace éclairé de la pièce, notamment dans l'axe de la fenêtre ou de la cheminée, pour la recherche de la lumière et de la chaleur. Mais quand la maison est pluricellulaire, une deuxième table est présente dans l'autre pièce; nous remarquons aussi sa présence dans les dépendances, la grange et le grenier. La table est donc un meuble plutôt réservé à l'espace domestique.

\section{Formes et fonctions de la table}

Intéressons-nous à ses fonctions et ses usages. La fonction d'un meuble dépend largement de sa forme. La table de ces paysans présente une particularité impliquant des usages propres. Nous trouvons fréquemment dans ces intérieurs la table à coffre : elle représente 95,84 \% des tables répertoriées. Les greffiers la désignent par divers termes ${ }^{30}$ et quelques inventaires donnent des renseignements sur les éléments la constituant. Ainsi un " couvercle " est très souvent mentionné : il s'agit d'une pièce d'un seul tenant à surface plane, qu'il faut vraisemblablement soulever pour atteindre le coffre. Cette façon de faire est la plus courante. Néanmoins une table creuse «à deux battants " a été recensée; là, il faut écarter les deux battants pour atteindre le caisson. Certaines tables sont dites jonchées (garnies de jonc). Mais cette mention ne signifie pas que la majorité d'entre elles soient garnies de ce matériau, cette notation a, au contraire, pu être faite pour son caractère exceptionnel. De même en ce qui concerne leur forme - rectangulaire, carrée? - rien ne permet de nous avancer. Ce qui est sûr malgré tout, c'est que certaines sont pourvues d'une serrure et d'une clé étant donné que quelques-unes sont décrites comme " table close sans serrure " ou " table sans fermeture ». Donc une table aux allures simples mais imposantes.

29. "Un châlit proche de la table " : Arch. dép. d'Ille-et-Vilaine, 4B 4170, Joseph Joubaud, 26 mai 1786, Activité?, Bains, 405 livres 16 sols. "Une couchette et une maie proche de la table " : Arch. dép. d'Ille-et-Vilaine, 4B 4172, Guillemette Hamon, 28 décembre 1788, Veuve, Bains, 1207 livres 14 sols.

30. Table creuse; table à pain; table close. 
Les fonctions de ces tables-coffres sont multiples. Il n'y a pas de doute qu'avec son couvercle la table est un meuble supportant et qu'avec son coffre elle devient un meuble contenant. C'est cette deuxième fonction que nous allons approfondir en recherchant ce que ces paysans peuvent bien y renfermer. Les greffiers ont le plus fréquemment relevé " une table creuse vide ", cependant, quelques rares tables ont livré leur contenu au moment de l'inventaire. Sur onze contenus relevés, neuf comportent de la vaisselle : une table " dedans deux petits plats d'étain, une assiette et un beurrier de faïence ${ }^{31} "$; une autre " table close sans serrure dedans deux assiettes d'étain, un petit plat de terre vernissée ${ }^{32}$ ". De même, nous trouvons des récipients relatifs à la crémerie, comme le beurrier, le pirot ${ }^{33}$ : une table dedans " trois poêles avec du lait, un pirotouer de terre de Fontenay, une vieille nappe de toile de brin réparon ${ }^{34}$ ". Une table nous a donné un contenu tout à fait intéressant : "Une table close sans fermeture dedans la moitié d'un pain de graisse et un jambon de lard fumé ${ }^{35}$. " Avec cet unique exemple, nous apprenons que la table peut servir de garde manger. Cela peut être une pratique étendue, ce qui expliquerait que bon nombre de celles-ci sont vides, car elles renfermaient des denrées périssables qui n'ont pas attendu l'inventaire. Un terme qui désigne cette table est également à prendre en compte ici, il s'agit de la " table à pain " ou encore " table à mettre le pain ". Il est vraisemblable alors que les paysans ont pour habitude de ramasser le pain dans cette table, voire de se servir de cette table comme pétrin pour préparer la pâte à pain. Cette table a donc des usages multiples.

D'autres tables ont une forme, une essence, une fonction particulière. Nous trouvons des tables faites de pierres et de palis, des tables longues, une table avec trois ou quatre "pots " qui peuvent être tournés, donc des tables dont le coffre a disparu et qui sont plus fines et raffinées. Dans ces intérieurs, les paysans ou plutôt leurs femmes, utilisent aussi des tables à buée qui servent, comme leur nom l'indique, à la buée, c'est-à-dire à la lessive, avec une inclinaison qui permet de frotter le linge.

\section{La table est-elle synonyme de sociabilité?}

Prendre connaissance des meubles "proches de la table " permet de saisir les habitudes quotidiennes. Ces meubles sont de deux types : des meubles contenants et des meubles à s'asseoir. Les contenants placés près de la table sont des coffres, des maies ou des bancs coffres. Cependant,

31. Arch. dép. d'Ille-et-Vilaine, 4B 4344, Pierre Debray, 14 août 1753, Activité?, Renac, 825 livres 10 sols.

32. Arch. dép. d'Ille-et-Vilaine, 4B 4375, Toussaint Garel, 21 juin 1770, Activité?, Sixt, 255 livres 16 sols.

33. Poêle à lait et pirot sont deux récipients dans lesquels on met le lait que l'on veut écrémer.

34. Arch. dép. d'Ille-et-Vilaine, 4B 4344, Robert Brulais, 14 août 1755, Activité?, Saint Just, 92 livres 13 sols.

35. Arch. dép. d'Ille-et-Vilaine, 4B 4344, Apposition de scellés, Jacques Launay, 31 juillet 1755, Activité?, Renac. 
cette disposition n'est pas généralisée, toutes les tables ne disposent pas de ce type de meubles dans leur environnement immédiat. Pour comprendre l'utilité de cette proximité, regardons de plus près ce que recèlent ces meubles. Malheureusement seuls trois meubles pour lesquels il est clairement indiqué qu'ils sont proches de la table révèlent leur contenu : " un banc coffre auprès de la table servant à mettre le lait ${ }^{36}$ "; " un grand coffre près de la table plein de bled ${ }^{37}$ "; " un coffre fermant de clef scellé proche de la table dedans quatre demés de bled noir, un linceul, un manteau à homme, un manteau à femme ${ }^{38}$ ". Trois meubles, trois contenus différents : des réserves céréalières, des produits laitiers et du linge; tout ce contenu n'est pas en lien direct avec la table. La plupart de ces meubles étant vides, nous ne pouvons que supposer les habitudes auxquelles ils renvoyaient. Peut-être sont-ils placés contre la table dans un souci de libérer de l'espace, et dans ce cas nous reprenons l'hypothèse de l'imbrication du mobilier, comme explication à cette association.

Pour savoir si les paysans se réunissent autour de la table, le meilleur indice est de repérer la présence de meubles à s'asseoir. Toutes les tables ne sont pas flanquées de ce type de meubles, là encore les sources restent imprécises, puisque pour 7,78 \% seulement des tables il est fait mention de cette association. Il s'agit pour 91,48 \% de bancs. Pour affiner ce chiffre : $48,93 \%$ de ces tables n'ont qu'un seul banc et $42,55 \%$ en ont deux. Les autres sièges, chaise, berchet, tabouret et billot n'ont été que rarement prisés autour de la table. Chez un paysan, nous trouvons " une table à pain avec deux bancs estimés 3 livres 10 sols " puis plus loin dans la liste " un billot au bout de la table estimé 5 sols $^{39}$ ". La logique veut en effet que les bancs soient placés contre les côtés les plus longs de la table et les autres sièges aux extrémités. Cette disposition des meubles autour de la table ne renvoie-t-elle pas, là aussi, à une hiérarchie par l'attribution du siège à telle personne selon son statut (chef de famille, enfant, convives, etc.)?

Finalement, nous pouvons nous demander pourquoi nous trouvons si peu de meubles à s'asseoir autour des tables. Certes, il y a à proximité le meuble marchepied du lit qui peut servir de siège. Mais, tout simplement, l'association table-sièges ne va peut-être pas de soi chez tous les paysans. Cette dernière hypothèse peut être confortée par la forme de la table. La table à coffre est basse et il est difficile de s'asseoir autour. Henri-François Buffet le constate : "La table était assez incommode, et quand on s'y asseyait, on ne savait pas où mettre les jambes ${ }^{40}$. "Une autre hypothèse

36. Arch. dép. d'Ille-et-Vilaine, 4B 4155, Mathurine Penhalleux, 21 février 1771, Veuve, gens de labeur, Notre Dame, 257 livres 18 sols.

37. Arch. dép. d'Ille-et-Vilaine, 4B 4152, Pierre Mahé, 18 septembre 1768, Laboureur, Saint Gorgon, 708 livres 10 sols.

38. Arch. dép. d’Ille-et-Vilaine, 4B 4091, Nicolas Macé, 2 décembre 1707, Activité?, Bains, 526 livres 6 sols.

39. Arch. dép. d'Ille-et-Vilaine, 4B 4167, Pierre Couë, 16 octobre 1783, Activité?, Notre Dame, 536 livres 18 sols.

40. BuffEt, Henri-François, En Haute Bretagne, coutumes et traditions d'Ille-et-Vilaine, des Côtes-du-Nord gallèses et du Morbihan gallo au XIXe siècle, Marseille, Laffite Reprints, 1982. 
voudrait que ces meubles soient placés ailleurs qu'autour de la table au moment où est dressé l'inventaire : "Un banc de table, une mauvaise chaise et un billot de bois estimés 1 sol auprès le feu ${ }^{41}$. " Ce serait alors un fait révélateur des gestes quotidiens et de la circulation des meubles dans l'espace domestique, au gré des nécessités. Ou bien tous les paysans n'ont pas dans leurs habitudes de s'attabler pour manger ou recevoir. Chacun se sert dans les plats posés sur la table, voire directement dans la marmite, et préfère soit rester debout soit s'asseoir sur la pierre de la cheminée ou ailleurs sur une chaise ou un banc-coffre. Ce que nous retiendrons de cette table si particulière c'est la combinaison de ses fonctions.

\section{Les sièges}

À l'époque qui nous occupe, le terme confort signifiait assistance, aide; il ne prit le sens de " tout ce qui constitue le bien-être matériel et les aisances de la vie ${ }^{42}$ " qu'au XIX ${ }^{\mathrm{e}}$ siècle. Dans les demeures paysannes, les habitants ne sont pas privés de ce confort. Ils recherchent une amélioration de leur bien-être et donc un mieux vivre qui se ressent dans l'organisation de leurs maisons. Ils le trouvent au travers des meubles à s'asseoir qui correspondent au confort rudimentaire qu'est la simple détente du corps. Ce type de meuble se présente sous diverses formes, la préférence accordée à l'un ou à l'autre type est révélatrice de la mentalité de leurs possesseurs.

De toutes les demeures visitées, 62,79 \% possèdent au moins un meuble à s'asseoir. Sur quoi s'assoit le paysan du pays de Redon? Au XVIII siècle, le banc, le banc-coffre et la chaise représentent à eux seuls 94 \% des sièges. Le banc-coffre est le meuble le plus constant au fil du siècle, face à la chaise qui progresse et au banc qui périclite. Les autres, en faible nombre, sont le tabouret, le billot, le berchet, l'escabeau et le fauteuil.

\section{Les différents types de sièges}

Les inventaires nous renseignement parfois sur l'aspect de ce mobilier. Le banc ne fait l'objet d'aucune description, mais on sait qu'il en existe de différentes tailles, certains étant qualifiés de "longs " et d'autres de " petits ". Un inventaire décrit un banc de cette façon : " une table creuse avec une planche servant de banc ${ }^{43}$ ", ce qui laisse penser qu'il s'agit d'un meuble très simple, dépourvu de toute ornementation. Sa valeur est modique, elle se situe au-dessous d'une livre. En ce qui concerne les chaises, là aussi les greffiers sont avares en descriptions, mais quelques caractéristiques peuvent être relevées, en particulier la matière de leur assise. Les plus nombreuses sont les chaises jonchées ou de jonc, peut-être parce

41. Arch. dép. d'Ille-et-Vilaine, 4B 4171, Julien Poirier, 24 mai 1787, Activité?, Bains, 448 livres 10 sols.

42. Dictionnaire de Littré, 1863.

43. Arch. dép. d'Ille-et-Vilaine, 4B 4103, Apposition de scellés, Jean Régent, 1720, Laboureur, Bains. 
que le jonc est une ressource naturelle abondante localement et de ce fait peu coûteuse. Viennent ensuite les chaises de bois, puis les chaises de paille ou " chaises ruchées de paille ». Ces dernières sont faites au moyen de paille de froment, de paille de seigle ou encore de paille de blé noir, là encore des ressources locales. Comme le banc, les chaises sont de diverses tailles, une seule a été qualifiée de " grande " et six autres de "petites". Certaines d'entre elles sont marquées d'une particularité, elles possèdent un coffre sous l'assise et ce coffre peut fermer à clef : "Une petite chaise de bois en forme de coffre fermant de clef et de clavette ${ }^{44}$." Quant aux bancs-coffres, aucune indication n'est apportée sur leur apparence, nous savons seulement qu'ils peuvent fermer à clef. Leur estimation est comprise entre 4 sols et 7 livres. Pour quelques-uns de ces trois sièges, les qualificatifs de " mauvais ", "vieux " sont utilisés, ils sont donc parfois dans un état d'usure avancée.

Pour ce qui est des autres sièges, ils sont tous d'une forme très sommaire à l'instar du billot, du berchet, de l'escabeau et du tabouret. Certains sont sûrement confectionnés par les mains du paysan lui-même, comme le billot qui n'est qu'une simple section d'un tronc d'arbre ${ }^{45}$. Par ailleurs, ils sont estimés à une valeur modique ${ }^{46}$. Seul le fauteuil, qui n'est présent que dans deux intérieurs, paraît plus élaboré. Pour Nicole de Reyniès ${ }^{47}$, il s'agit d'un siège d'une place à dossier et accoudoirs; cela fait de lui un siège plutôt confortable par rapport aux autres. Un inventaire mentionne " un fauteuil en bois estimé 3 livres ", il a donc une valeur non négligeable. Du fait qu'il n'est signalé que dans des inventaires de la fin du siècle, nous pouvons penser que c'est un meuble qui apparaît. Ces meubles peuvent être qualifiés de secondaires : nous les rencontrons dans des maisons où se trouvent déjà un banc-coffre, une chaise ou un banc.

Sans nul doute, tous ces meubles à s'asseoir frappent par leur aspect fruste. Le paysan semble ne s'offrir qu'un confort relatif car il s'assoit sur du dur, aucun siège n'étant rembourré. Comment se répartissent les trois principaux sièges dans les maisons?

44. Arch. dép. d'Ille-et-Vilaine, 4B 4344, Joseph Prévaire, 2 octobre 1755, Laboureur, Renac, 148 livres 3 sols.

45. L'escabeau se présente comme " un siège d'une place sans dossier dont le dessus est plein, il est monté sur pied ou sur panneaux verticaux " (REYNIES, Nicole de, Le mobilier domestique, vocabulaire typologique, Paris, Imprimerie Nationale, 1987, tome 1). Le berchet et le tabouret se présentent sous la même forme : ni dossier, ni accoudoirs.

46. Le billot n'a été estimé qu'une fois seule à 5 sols; Le berchet coûte moins de 5 sols; La valeur du tabouret est comprise entre 2 sols et 1 livre; et l'escabeau, n'étant jamais estimé, coûte moins de 5 sols.

47. REYNIES, Nicole de, Le mobilier domestique, vocabulaire typologique, Paris, Imprimerie Nationale, 1987, tome 1. 
Tableau 2 - Fréquence des trois principaux meubles à s'asseoir dans les foyers

(68,42\% des 691 foyers étudiés n'ont aucune chaise pour la période 1700-1730)

\begin{tabular}{|c|c|c|c|c|c|c|}
\hline & $\begin{array}{c}\text { Nombre par foyer } \\
\text { 691 cas soit 100 \% }\end{array}$ & 0 & 1 & 2 & 3 & 4 et plus \\
\hline \multirow{1}{*}{$1700-1730$} & Chaises & $68,42 \%$ & $21,05 \%$ & $6,31 \%$ & $4,21 \%$ & 0 \\
& Bancs-coffres & $49,48 \%$ & $45,26 \%$ & $4,21 \%$ & $1,05 \%$ & 0 \\
& Bancs & $71,42 \%$ & $18,36 \%$ & $9,18 \%$ & $1,02 \%$ & 0 \\
\hline $1731-1760$ & Chaises & $63,87 \%$ & $19,33 \%$ & $8,40 \%$ & $2,10 \%$ & $6,30 \%$ \\
& Bancs-coffres & $67,83 \%$ & $31,68 \%$ & $0,49 \%$ & 0 & 0 \\
& Bancs & $87,52 \%$ & $6,01 \%$ & $5,55 \%$ & $0,92 \%$ & 0 \\
\hline $1761-1790$ & Chaises & $70,57 \%$ & $13,96 \%$ & $7,92 \%$ & $4,90 \%$ & $2,64 \%$ \\
& Bancs-coffres & $69,59 \%$ & $26,57 \%$ & $3,49 \%$ & $0,35 \%$ & 0 \\
& Bancs & $92,92 \%$ & $4,10 \%$ & $2,61 \%$ & $0,37 \%$ & 0 \\
\hline
\end{tabular}

À la lecture de ces chiffres, nous constatons une diminution de ces meubles (augmentation du pourcentage des inventaires faisant état d'aucun de l'un de ces trois meubles), mais chacun de manière différente. Le banc-coffre est le meuble le plus implanté dans ces maisons. Au début du siècle la moitié des foyers en possèdent un mais, par la suite, il recule fortement. Comment expliquer cette préférence pour le banc-coffre? Outre son office de meuble à s'asseoir, il sert également comme meuble de rangement et de marchepied quand il est accolé au lit. De ce point de vue il est représentatif des attentes des paysans face à leur mobilier : il combine plusieurs fonctions ce qui sur le plan de l'organisation de l'espace évite l'encombrement. Le banc, quant à lui, déserte également les intérieurs sur la période. Au début du siècle il est déjà absent dans environ les trois-quarts des foyers, puis il disparaît quasiment. La chaise connaît des évolutions divergentes (déclin puis progression) : nous la trouvons dans le tiers des foyers environ.

Bien que la plupart des maisons ne comptent qu'un seul de ces sièges, la coexistence entre eux n'est pas non plus chose rare. Le modèle de coexistence est le banc-coffre et la chaise, donc un meuble lourd et fixe avec un meuble plus léger et mobile. Quelques demeures paysannes ont le privilège d'avoir un échantillon plus varié, comme le montre cet exemple : "Un banc coffre prisé 4 livres, [...], trois chaises de paille, un banc et deux billots prisés le tout 10 sols ${ }^{48}$. "Toutes les maisons des paroisses du Pays de Redon ne disposent pas du même nombre de sièges. Celles des paroisses les plus éloignées de la ville de Redon en comptent moins.

\section{L'usage des sièges}

Maintenant que nous savons que les sièges sont présents dans 62,79\% des maisons, nous allons nous intéresser aux activités au cours desquel-

48. Arch. dép. d'Ille-et-Vilaine, 4B 4291, Yves Bouvneuf, 13-14 avril 1757, Fégréac, 405 livres 7 sols. 
les les paysans s'assoient. Les indices sur lesquels nous nous appuyons sont l'emplacement de ces sièges dans la maison, le contenu de certains d'entre eux, l'association avec des outils de la vie quotidienne. Quand ils entourent la table, ils témoignent de la sociabilité au moment du repas. Placés devant les lits, notamment les bancs-coffres, ils servent de marchepied, ou à ranger des effets, ou encore à s'asseoir (à table?). Ils sont placés aussi autour du pôle principal de la demeure, la cheminée, comme ces exemples permettent de l'avancer : "deux billots de bois à côté du foyer estimé 4 sols $^{49}$ "; " une grande chaise de paille dans un coin de la cheminée estimée 1 livre 10 sols $^{50}$ "; " une chaise de bois proche le foyer estimée 4 livres ${ }^{51}$ ". La cheminée est en effet l'endroit de la maison le plus éclairé et aussi le plus chaud. Nous avons remarqué que c'est la chaise qui est privilégiée pour cet emplacement, sûrement parce que la chaise est facilement déplaçable. Peut-être les paysans parlent-ils mais, même assis, ils s'occupent les mains. Dans de nombreux intérieurs, des rouets et encore des travouils sont placés près de la cheminée : l'activité du filage, réservée aux femmes, se fait donc assise devant la cheminée. Les paysans ont aussi des sièges dans les bâtiments annexes comme le suggèrent ces exemples : "trois chaises dans l'étable de la maison estimées 15 sols ${ }^{52}$ "; " deux chaises de jonc dans l'étable ${ }^{53}$ ". Ces chaises, les paysans les utilisent sûrement au moment de la traite des vaches. Ils préfèrent atténuer la pénibilité du travail en adoptant la position assise au lieu de s'accroupir ou de se courber. Nous rencontrons une chaise dans un cellier qui abrite, avec les instruments de transformation de fruits en boissons, les réserves de vin et de cidre. Cette chaise peut servir comme meuble de " confort " lors d'un travail, ou comme élément de sociabilité. Les inventaires précisent aussi les usages de ces sièges par les objets qui leur sont associés lors de la prisée. Ainsi nous avons relevé des bancs avec leurs braies, et encore des bancs avec leur égrugeoir. En effet certains greffiers précisent l'usage du banc en indiquant soit "banc à s'asseoir " ou " banc avec tel outil ". Ces deux outils, braie et égrugeoir, sont posés sur le banc et ils servent aux premières étapes de la fabrication de fils. Nous avons également trouvé " un tabouret avec un dévidoir "; ce dernier, comme le travouil, est un instrument servant à mettre en écheveau le fil qui est sur le fuseau.

49. Arch. dép. d'Ille-et-Vilaine, 4B 4155, Guillaume Guillemé, 8-9 novembre 1771, Laboureur, Bains, 913 livres 13 sols.

50. Arch. dép. d'Ille-et-Vilaine, 4B 4095, Renée Lejeune, 20 août 1711, Veuve, Avessac, 372 livres 3 sols.

51. Arch. dép. d'Ille-et-Vilaine, 4B 4102, Apposition de scellés, Jean Rape, 19 janvier 1718, Laboureur, Notre Dame.

52. Arch. dép. d'Ille-et-Vilaine, 4B 4155, François Devaux, 22 juin 1771, Activité?, Notre Dame, 201 livres 14 sols.

53. Arch. dép. d'Ille-et-Vilaine, 4B 4291, Guillaume Renaud, 26 juillet 1764, Activité?, Avessac, 437 livres. 


\section{Les chaises remplacent progressivement les bancs}

Les meubles nous renseignent également sur les mentalités des paysans. Le rapport de ces derniers avec leurs meubles, leur préférence pour tel meuble plutôt que pour tel autre, peut nous expliquer comment ils choisissent d'organiser leur intérieur. Prenons le cas de la chaise et du banc ${ }^{54}$. Tous deux ont la même fonction mais ont des contraintes différentes sur les habitants et sur l'espace. L'un va être privilégié par rapport à l'autre, nous allons tenter de comprendre ce phénomène.

Tableau 3 - Répartition des chaises et des bancs dans les foyers

(en pourcentage du nombre de foyers)

\begin{tabular}{|c|c|c|c|c|}
\hline & $\mathbf{1 7 0 0 - 1 7 3 0}$ & $\mathbf{1 7 3 1 - 1 7 6 0}$ & $\mathbf{1 7 6 1 - 1 7 9 0}$ & ensemble \\
\hline Chaises & $29,66 \%$ & $50,88 \%$ & $52,37 \%$ & $47,85 \%$ \\
Bancs & $27,59 \%$ & $15,87 \%$ & $9,78 \%$ & $15,60 \%$ \\
\hline
\end{tabular}

Au début du siècle, il y a autant de chaises que de bancs. Par la suite, à partir de la moitié du siècle, la chaise se démarque largement. Le banc connaît une régression importante. La chaise investit tout d'abord les foyers où le montant de l'inventaire est assez élevé et, à la fin de la période, elle est présente dans des intérieurs plus humbles. Autre fait notable, la présence de la chaise est plus importante dans les maisons paysannes des faubourgs de la ville, qui en possèdent deux ou plus, alors que celles des autres paroisses proches de la ville en comptent au maximum deux et les intérieurs des paroisses plus éloignées en sont presque totalement dépourvus. Comment expliquer cette victoire de la chaise sur le banc?

La progression de la chaise sur le banc, c'est la confrontation de la mobilité et de la fixité, de l'individuel et du collectif. Au cœur même de cette confrontation, c'est une nouvelle prise en compte de l'espace et du corps. Entre les deux sièges, le confort est différent; tous deux permettent au corps, même dans une position verticale, un repos musculaire, par la décontraction des jambes. Cependant avec le banc, le corps reste tassé alors qu'avec la chaise, par l'appui du dos sur le dossier, le corps tout entier se décontracte et se relâche, ce qui autorise un confort plus appréciable. Ensuite la mobilité est autre. Le banc est un meuble lourd avec une forme longue, par conséquent il est encombrant. C'est ainsi que la plupart des bancs sont accolés à la table et donc restent fixes. Tandis que la chaise, de par son poids et sa taille, est plus facile à déplacer au gré des besoins et nécessités. Daniel Roche le souligne, " la chaise permet de recomposer l'espace en fonction des rythmes d'utilisation, autour de l'âtre, de la table ${ }^{55}$ ". Cet exemple où " trois chaises [sont] dans un petit cabinet au derrière de la demeure ${ }^{56}$ "

54. Nous laissons de côté le banc-coffre car il est plus qu'un simple meuble à s'asseoir.

55. Roche, Daniel, Histoire des choses banales, naissance de la consommation aux XVII XIX siècles, Paris, Fayard, 1997.

56. Arch. dép. d'Ille-et-Vilaine, 4B 4375, Mathurin Rollais, 5 octobre 1755, Activité?, Sixt, 374 livres 10 sols. 
pourrait conforter l'hypothèse qu'en cas de non-utilisation, elles sont entreposées dans une sorte de débarras. Une autre explication est plus axée sur les mentalités, mais inhérentes à l'organisation de l'espace : c'est le passage du meuble de groupe à un meuble individualisé. Le banc se caractérise par une façon de s'asseoir collective où les corps s'alignent côte à côte, ce qui implique une proximité. Avec la chaise, c'est une nouvelle relation avec son corps et celui des autres. À présent, chacun occupe "son " siège et par conséquent il s'impose un espace propre.

\section{Ordre et désordre des foyers}

Pour finir cette étude sur l'organisation intérieure de ces maisons paysannes, nous allons nous pencher sur le rangement. Dans les inventaires, même des plus humbles, nous voyons que les paysans s'entourent d'objets et d'effets. Reste à savoir comment ces paysans les rangent et s'ils ont le souci de l'ordre. Notre attention va se tourner principalement vers les meubles contenants qui répondent à cette nécessité. Dans ces intérieurs, quatre principaux meubles de rangement se dégagent. Le coffre est le plus présent, il représente $55,42 \%$ des contenants, il est suivi par la maie $(22,99 \%)$, l'armoire (10,28 \%), et le banc-coffre (7,67 \%).

\section{Les meubles de rangement}

Le coffre a-t-il une particularité dans cette région? Il se décline en plusieurs dimensions lorsque les adjectifs " petit ", " moyen " ou " grand " lui sont attribués. C'est un meuble se fermant par le dessus au moyen d'un couvercle, qui n'est pas toujours présent, et qui ferme à clef, même si tous ne sont pas suivis de la mention "fermant de clef et clavette". Du fait du laconisme des sources, la description physique du coffre est malaisée. Il semble cependant qu'il soit de forme simple. L'un d'entre eux a cependant attiré notre attention; il est décrit comme « un ancien coffre fermant de clef et clavette avec deux tiroirs dans le bas sans clef ${ }^{57}$ ". Quant à leur état, beaucoup sont qualifiés de vieux, quelques-uns de méchants, éffoncés, mauvais et très rarement de neuf. Quant à sa valeur, elle est variable, les deux estimations extrêmes repérées sont de 5 sols à 14 livres.

Pour désigner la maie, les greffiers usent de divers termes ou expressions précieuses. Même si le mot " maie " est le plus utilisé, nous avons repéré " maie à pétrir " et " maie à faire le pain " dans les inventaires allant de 1700 à 1730, " maie à faire la pâte " dans les années 1760. Ces expressions nous indiquent que ce meuble creux servait à la fabrication du pain. Le terme est-il resté ou cette pratique existait-t-elle encore? Il semblerait qu'elle soit toujours avérée chez certains paysans puisque la maie est prisée avec sa tourtière ${ }^{58}$. Une autre dénomination renvoie à un autre usage,

57. Arch. dép. d'Ille-et-Vilaine, 4B 4344, Jolly, 15 mars 1756, Activité?, Sixt, 445 livres 14 sols 6 deniers.

58. Dans les inventaires le terme relevé est "antourtière ". Il s'agit d'un instrument qui sert à faire cuire la tourte c'est-à-dire le pain rond. Celle-ci est faite dans de la pierre. 
il s'agit de la " maie à piller les pommes ". Quant à son apparence, nous pouvons l'imaginer en remarquant que l'élément mis en avant lors de sa description est son couvercle. Elle peut aussi fermer à clef. Comme pour d'autres meubles, beaucoup sont dites "vieille ", " méchante ", "éffoncée " et " mauvaise".

Pour l'armoire, il existe aussi des dimensions variées, bien qu'un grand nombre d'entre elles soient dites "petites ». Les greffiers utilisent aussi l'expression " demie-armoire ", voire " petite demie-armoire ». Le modèle le plus fréquent sur toute la période est l'armoire à deux battants. Mais il existe des armoires à un, trois et quatre battants. Sur leur façade se comptaient non seulement des battants mais aussi des tiroirs et des tirettes ${ }^{59}$.

Ces meubles semblent donc se caractériser par la simplicité de leur facture mais aussi par leur état d'usure avancée. Même très abîmés, ils ne sont pas renouvelés. Comment ces meubles investissent-ils les maisons?

\section{Tableau 4 - Évolution des quatre meubles de rangement}

(en pourcentage du nombre de meubles contenants - Ex. = 62,27\% des meubles contenants répertoriés sont des coffres pour la période 1700-1730)

\begin{tabular}{|c|c|c|c|c|}
\hline & $\mathbf{1 7 0 0 - 1 7 3 0}$ & $\mathbf{1 7 3 1 - 1 7 6 0}$ & $\mathbf{1 7 6 1 - 1 7 9 0}$ & Ensemble \\
\hline Coffre & $62,27 \%$ & $58,96 \%$ & $50,00 \%$ & $55,42 \%$ \\
Maie & $18,77 \%$ & $21,29 \%$ & $25,91 \%$ & $22,99 \%$ \\
Armoire & $5,39 \%$ & $8,88 \%$ & $13,18 \%$ & $10,28 \%$ \\
Banc-coffre & $10,22 \%$ & $7,43 \%$ & $6,98 \%$ & $7,67 \%$ \\
\hline
\end{tabular}

Chacun de ces quatre meubles suit une évolution différente dans ces maisons. Le coffre et le banc coffre reculent alors que l'armoire et la maie investissent les intérieurs. Comment expliquer ce phénomène?

Le coffre est fortement intégré dans la vie quotidienne des paysans. De 1700 à 1730, il est présent dans $100 \%$ des foyers, de 1761 à 1790 dans $98 \%$. Nous pouvons encore mieux apprécier son enracinement avec l'examen du nombre de coffres par foyer. Ainsi au début du siècle $31 \%$ des foyers comptent trois coffres, $21 \%$ deux et $20 \%$ quatre. À la fin du siècle, $32 \%$ des foyers en possèdent deux, $25 \%$ un seul et $21 \%$ trois. De ce fait, nous pouvons avancer que le coffre fait partie intégrante du mobilier et du quotidien des paysans. Il occupe divers emplacements dans les maisons. Un paysan de la paroisse Notre-Dame de Redon possède « un coffre de bois de chêne au bout du lit, un grand coffre avec sa clef et clavette à main droite comme on entre, un petit coffre de bois avec sa clef dans l'allée ${ }^{60} "$. Il est très souvent placé près du lit ou des ouvertures (surtout des portes). L'entrée de la maison est aussi un lieu privilégié, un coffre y est quasiment toujours disposé. Il trouve également place dans les dépendances, grenier,

59. Une tirette est une tablette à glissière adaptée à certains meubles.

60. Arch. dép. d'Ille-et-Vilaine, 4B 4105, Olivier Rape, 29 mars 1721, Laboureur, Notre Dame, 866 livres 13 sols. 
étable, grange, appentis et cellier, parfois dans des endroits plus particuliers comme " un coffre derrière l'armoire ${ }^{61}$ ". Le coffre investit tous les espaces de l'habitat et de son environnement, puisque nous avons rencontré " un petit coffre à 5 sols dans le jardin ${ }^{62}$ ".

La maie, déjà bien intégrée dans les foyers, s'enracine plus profondément. De 1700 à 1730, 78 \% des foyers en possèdent au moins une, alors que de 1760 à 1790 , ce sont 91 \% des foyers. Combien de maies par foyer? Au début du siècle, 59 \% des intérieurs sont équipés d'une maie, 22 \% n'en ont pas et $15 \%$ seulement en comptent deux. Dans les dernières décennies, seuls $9,3 \%$ des foyers en sont dépourvus, $57 \%$ en ont une, $26 \%$ deux. Tous ces chiffres montrent son intégration. Dans une grande majorité des cas, les paysans la placent devant un lit. De petite taille par rapport au coffre, elle occupe peu d'espace et allie aussi bien la fonction de marche-pied que de meuble de rangement et, pourquoi pas, de meuble à s'asseoir. Les paysans l'apprécient car c'est un meuble multifonctionnel et peu encombrant. C'est un meuble qui peut occuper divers endroits, ce qui est un avantage certain. Autre avantage, sa valeur, comprise entre 6 sols et 12 livres. Les intérieurs les plus modestes peuvent se permettre de l'accueillir.

L'armoire s'incruste assez rapidement. De 1700 à 1730, elle est présente dans $20,79 \%$ des foyers et de 1761 à 1790 dans 47,31 \%. Au début du siècle, $16 \%$ des foyers ont une armoire, à la fin de la période $38 \%$ en ont une et 7,9\% deux. Son emplacement dans la maison est assez flou. Elle fait partie de la liste de biens contenus dans l'embas principal et se place très souvent près d'un lit. Peut-être sert-elle alors de cloison pour procurer un minimum d'intimité. Toutefois certains paysans la placent dans l'entrée de la maison, endroit éclairé et lieu de passage obligé pour tous les visiteurs : les regards sont donc orientés vers elle. Un seul cas nous indique qu'une armoire est placée dans un cellier. L'armoire est un meuble qui n'est pas placé n'importe où, ce qui révèle son prestige et son importance aux yeux de ses possesseurs. Quant à sa valeur, elle est comprise entre 10 sols et 45 livres, ce qui fait d'elle un meuble aussi coûteux que le lit.

Attardons-nous sur la confrontation coffre-armoire. Dans la symbolique, ce sont deux meubles antagonistes, le coffre est synonyme d'errance et l'armoire de stabilité. Le constat général est que les plus humbles privilégient toujours le coffre alors que l'armoire entre dans les maisons les plus aisées. De ce fait, ce dernier meuble serait un signe extérieur de richesse puisqu'il n'est pas à la portée de toutes les bourses. Néanmoins, certains paysans font coexister ces deux meubles dans leur intérieur. Il est intéressant dans ce cas de comprendre comment ils les répartissent dans l'espace. Il ressort qu'à travers plusieurs exemples ${ }^{63}$, dans lesquels la maison est

61. Arch. dép. d’Ille-et-Vilaine, 4B 4165, Jean Duchesne, 19 octobre 1781, Activité?, Saint Gorgon, 246 livres 10 sols.

62. Arch. dép. d'Ille-et-Vilaine, 4B 4160, Fabien Bouvier, 26-29 novembre 1776, Laboureur, Bains, 662 livres 5 sols.

63. Exemple : dans la pièce principale une armoire prisée 25 livres et dans une petite chambre à côté un grand coffre prisé 8 livres (Arch. dép. d'Ille-et-Vilaine, 4B 4152, Denis 
pluricellulaire, le coffre est relégué dans la ou les pièces secondaires. L'armoire reste toujours dans la pièce principale, c'est-à-dire la pièce où l'on vit et reçoit, ce qui conforte l'idée que les paysans voient leur armoire comme un meuble de prestige. Mais il faut garder à l'esprit que l'armoire est encore loin d'avoir détrôné le coffre car celui-ci reste l'apanage de la quasi-totalité de ces paysans du fait de sa taille et de son coût.

\section{Les stratégies de rangement}

Que nous ayons à faire à l'un ou l'autre de ces meubles, le mode et la stratégie de rangement n'est pas la même. À première vue, le coffre, tout comme le banc-coffre et la maie, suppose l'entassement pêle-mêle des effets, tandis que l'armoire suppose un rangement plus méthodique. Là, la confrontation prend tout son sens. Soulevons le couvercle du coffre, banccoffre ou maie et ouvrons les battants de l'armoire pour découvrir ce que ces paysans y renfermaient et leurs façons de ranger. Rien que par cette phrase nous percevons que la posture face à ces meubles n'est pas la même pour y chercher quelque chose. Pour chercher dans un coffre, il faut se courber alors que face à l'armoire, le corps reste plus ou moins droit. Que rangent ces paysans dans ces meubles fermés et comment?

À travers les exemples rencontrés nous remarquons, par la longue liste des effets cités, la grande capacité des coffres. Mais chaque coffre a t-il un contenu spécifique ou diversifié? Quand le coffre est le seul meuble contenant du foyer son contenu est hétéroclite ${ }^{64}$. Y sont empilés des vêtements, du linge, des céréales, des papiers, des aliments. Il semblerait que, quand plusieurs coexistent dans la même maison, chacun ait un contenu bien spécifique, certains sont réservés aux grains et d'autres aux vêtements, linge, papiers. Il semblerait également que chaque membre de la famille, ou un regroupement de membres, ait son coffre pour y ranger ses effets. Quant aux stratégies de rangement à l'intérieur du coffre, il est très malaisé de s'avancer, car chaque individu crée ses habitudes. Cependant, à la lecture

Macé, 20 avril 1769, Activité?, Bains, 318 livres 18 sols); Dans une pièce principale une armoire et dans une chambre de l'autre côté trois coffres : (Arch. dép. d'Ille-et-Vilaine, 4N 4091, Apposition de scellés, Denise Chevallier, 8 mars 1707, Activité?, Bains).

64. Arch. dép. d'Ille-et-Vilaine, 4B 4344, Jolly, 15 juin 1756, Activité?, Sixt, 445 livres 14 sols 6 deniers : "Un coffre ancien fermant de clef et clavette avec deux tiroirs dans le bas sans clef dans lequel deux coiffes et deux collerettes de toile déliée; Deux coiffes et quatre mouchoirs de gros brin; Six coiffes (une garnie de dentelle), sept collerettes de pareille toile; Une vieille brassière d'étamine noire; Une vieille brassière de penchinat] d'Amboise; Une jupe de serge avec de la toile devant; Un manteau à femme de ras noir doublé de vieux castor; Un tablier de toile de brin percé en différents endroits; Une mauvaise jupe de toile rallongée; Un tablier de toile de brin; Une brassière de serge brune; Une vieille brassière de toile de brin; Une vieille jupe de ras noir; Un mauvais tablier de ras noir; Seize mauvaises coiffes et treize mauvais collets de toile de brin; Quatre mauvaises chemises à l'usage de la feue [défunte] de toile de brin reparon; Une vieille nappe de toile de brin d'environ dix empans à mettre sur les corps; Un petit pochon dans lequel environ une godelée et demie de graine de chanvre; Une petite potée de beurre fort de cinq livres; Une potée de saindoux de quatre livres ; Une vieille paire de souliers à l'usage de femme. " 
de la liste des contenus, nous nous apercevons que les effets sont regroupés par catégorie. Nous ne trouvons pas une potée de beurre au milieu des vêtements, nous pouvons donc penser que, dans le coffre, chaque catégorie est regroupée dans un coin et empilée. On remarque aussi l'utilisation de sacs pour contenir certains effets : la poche, qui n'est autre qu'un sac de toile, renfermant les différents bleds; le pochon qui contient l'argent du couple; des paniers dans lesquels les femmes mettent leurs accessoires de coiffure; plus rarement sont utilisées des boîtes.

Les bancs-coffres, souvent placés devant les lits, renferment généralement des vêtements ou des céréales. Les maies, quand elles ne servent pas de pétrin, contiennent des effets divers, des récipients à crémerie, de la vaisselle, des vêtements et même des céréales. Bien qu'elles puissent être d'un usage à rangement spécifique, elles deviennent, chez certains paysans, de vrais fourre-tout.

Avec l'armoire, comme nous l'avons vu plus haut, c'est la rationalisation des gestes qui prédomine (le corps reste debout); mais y a t-il une rationalisation du rangement? Bien que le recours à l'empilement soit plausible, le rangement par tri est largement suggéré. La grande majorité des paysans ont des armoires dont le contenu est identique à celui du coffre, c'est dire des vêtements, du linge, des papiers, de l'argent, voire des réserves alimentaires. Quant à savoir comment s'effectue le rangement à l'intérieur, il est quasiment impossible de s'avancer. Les sources sont imprécises car le contenu est donné sous forme de liste sans autre indication.

Quand coexistent dans un même foyer une armoire et un coffre, qu'est ce que les paysans préfèrent mettre dans l'un et dans l'autre? Nous avons remarqué que, dans certains foyers, l'armoire referme les " habillements " d'homme, et ceux de femme sont rangés dans un coffre. Cette constatation n'est pas à généraliser car, plus fréquemment, hormis les réserves céréalières, uniquement réservées aux coffres, le contenu du coffre et celui de l'armoire sont identiques. Cette confrontation entre le coffre et l'armoire est vraisemblablement autant un indice de richesse que la recherche de la rationalisation de gestes et du rangement. Même si ces contenants peuvent devenir un vrai fourre-tout, les paysans optent tout de même pour une stratégie de rangement par le recours de sacs, paniers pochons pour séparer chaque catégorie d'effets.

Outre les quatre principaux contenants, d'autres meubles ont également une fonction de rangement (3,64 \%). Il s'agit du garde manger, du vaisselier, du comptoir-dressoir, du buffet, du bahut, de la huche et de la presse, qui n'investissent qu'un nombre limité de foyers, puisque sur toute la période seulement $14 \%$ de foyers en sont pourvus. Mais tous n'ont pas la même importance aux yeux des paysans. La paire de presse n'est recensée que dans une seule maison et au début du siècle. C'est un meuble imposant, contenant " les hardes de la veuve ", qui était placée dans l'allée et donc à l'endroit le plus exposé aux regards. Même sa valeur n'est pas négligeable car elle est estimée 10 livres. La huche est présente dans six inté- 
rieurs inventoriés au début du siècle puis disparaît des inventaires, l'une d'entre elles renfermait " les hardes de la servante". Quant au bahut, qui tient sa forme du coffre lui aussi, est aussi effacé que la huche (4,06\%). Ces trois meubles semblent déserter les maisons au fil du siècle voire même disparaître. Les autres meubles connaissent quant à eux une progression. À l'instar du buffet $(0,41 \%)$ qui sert le plus souvent à supporter un vaisselier. Le garde-manger ( $0,80 \%)$ sert à entreposer de la vaisselle, de la crémerie. Le vaisselier (1,01\%), posé soit sur un buffet ou un comptoir, contient surtout des écuelles, assiettes, plats. Le comptoir-dressoir (1,09\%) forme un meuble à deux corps dissociables. Le dressoir a le même usage que le vaisselier. Le comptoir renferme surtout des habits. Comme nous l'avons constaté, 86 \% des foyers de l'échantillon ne possèdent pas ces genres de contenants. Grâce à leurs contenus, ou à la forme de quelques-uns, nous comprenons pourquoi les paysans les délaissent par rapport aux coffres, maies ou armoires. En fait, ils renferment les mêmes objets et effets que les quatre principaux. Nous notons que les paysans privilégient les meubles qui permettaient de ranger un maximum de choses, de natures différentes. Les derniers meubles étudiés, surtout le vaisselier et le comptoir, répondent à un usage spécifique qui ne correspond pas encore aux attentes des paysans. La spécialisation des contenants n'est pas encore leur priorité, la promiscuité de la pièce unique en est la principale raison.

Ce que nous avons constaté lors de l'étude des modes de rangement de ces maisons c'est que l'ordre qui y règne est soumis à la hiérarchisation des objets. En effet, certains objets ou effets sont rangés dans un meuble alors que d'autres, d'usage courant et de peu de valeur, semblent plutôt joncher le sol ${ }^{65}$. Un rapport s'établit donc entre l'homme et l'objet. C'est ainsi que les vêtements, chers et nécessaires, sont les principaux effets contenus dans le coffre ou l'armoire. Au même titre, l'argent et les papiers de la famille sont enfermés.

Cette étude a tenté de découvrir les différents meubles que les paysans du Pays de Redon placent dans leurs maisons. Ils répondent essentiellement, voire uniquement, à des besoins physiques : dormir, manger, se détendre. Outre cette réponse aux besoins élémentaires, le souci du rangement est aussi important dans ces maisons, même s'il semble se dégager un désordre apparent. Les deux principaux meubles, par conséquent indispensables, sont le lit et le coffre.

Les caractéristiques majeures de ce mobilier paysan sont tout d'abord sa multifonctionnalité. Elle est due au fait que la quasi-totalité des paysans vit dans une seule pièce qui doit réunir toutes les fonctions. Cette particularité permet de gagner de la place ou du moins d'éviter l'encombrement. La distribution du mobilier se fait autour des pôles de la pièce : la cheminée, les ouvertures et le lit (qui constitue un espace à lui seul). Le second

65. Tous les objets prisés ne sont pas renfermés dans un meuble. Les ustensiles de cuisine sont le plus souvent placés à même le sol, dans la cheminée ou auprès. 
trait remarquable est l'imbrication des meubles entre eux. Multifonctionnalité et imbrication permettent une meilleure circulation de tous dans l'espace domestique. La promiscuité impose aussi de vivre sous le regard des autres; la façon dont sont distribués ces meubles est un moyen de rechercher un semblant d'intimité, comme en témoigne la pratique de disperser les lits dans l'espace habité ou dans les dépendances.

En ce qui concerne les meubles en eux-mêmes, ils semblent pour beaucoup dans un état d'usure avancée, la pérennité du meuble était de rigueur. De même, leur facture semble simple, ils sont dépourvus d'ornementation, ce qui révèle que la priorité était leur fonctionnalité. Ces paysans ont donc un mobilier qui doit être à la fois utile, robuste et au moindre coût. D'ailleurs le renouvellement semble rare, vu le peu de meubles dits neuf. L'impression générale qui se dégage de ces intérieurs est la pauvreté dans laquelle vivaient une grande majorité de ces paysans.

\section{RESUME}

La maison rurale et surtout son intérieur nous apprennent énormément sur les mentalités, les façons de vivre et d'habiter, des paysans. Le mobilier étant un élément majeur dans l'organisation de cet espace domestique, son étude permet comprendre le rapport entre le meuble et son possesseur et le rapport entre le meuble et l'espace qu'il occupe. Ceci révèle à la fois des habitudes (entre autre une hiérarchisation des dormeurs) et des évolutions (passage du banc à la chaise ou du coffre à l'armoire). Dans ces intérieurs très modestes le mobilier est le reflet deux principales contraintes : la promiscuité et la pauvreté. Il se caractérise par sa multi-fonctionnalité et sa durabilité.

\section{ABSTRACT}

Rural house and especially her interior, privileging the daily gestures, learn to us enormously on the mentalities, the living manners and the occupying manners, of this countrymen. The furniture, being an major element in the organization of this home space, his study is interesting of an double point of view: to apprehend the relation between a piece of furniture and his owner, and relation between piece of furniture and the space that it's occupied. It's divulged the uses (among other things an sleepers' hierarchization), and the change of mentalities by the clue of the passage from bench to chair or from chest to wardrobe. It's proving that in this interiors very modest furniture is the reflection of two mains constraints: promiscuity an poverty. So, it's characterizing by his multi-functionality and his span of life. 
